\title{
Naphthalene Compound
}

National Cancer Institute

\section{Source}

National Cancer Institute. Naphthalene Compound. NCI Thesaurus. Code C54679.

A class of compounds with the basic naphthalene structure, an aromatic hydrocarbon compound, consisting of two fused benzene rings. 